\title{
Comparative Study on the Compressive Strength of a New Ceramic Reinforced Glass lonomer (Amalgomer CR) and Resin-coated High Strength Glass lonomer Cement (Equia Forte) with a Nanohybrid Composite Material (Tetric N Ceram) in a Simulated Oral Environment: An In Vitro Study
}

${ }^{1}$ Sonu Baby, ${ }^{2}$ Afin Ummar, ${ }^{3}$ Josey Mathew, ${ }^{4}$ Liza George, ${ }^{5}$ Sinju Paul

\begin{abstract}
Aim: To evaluate and compare the compressive strength of a new ceramic-reinforced glass ionomer (Amalgomer CR) and resin coated high strength glass ionomer .cement (GIC) (Equia forte) with a nanohybrid composite (tetric $\mathrm{N}$ ceram).
\end{abstract}

Methodology: Twenty four maxillary premolar teeth were selected. Selected teeth after cleaning were mounted in acrylic resin blocks exposing the crown. Class II cavities were prepared. Samples were divided into three groups of 8 teeth;

- Group 1: Class II cavity restored with Tetric N Ceram composite (control group)

- Group 2: Class II cavity restored with amalgomer CR

- Group 3: Class II cavity restored with equia Forte

Restored samples stored in artificial saliva for 2 weeks and subjected to compressive strength test using the universal testing machine at a cross head speed of $0.5 \mathrm{~mm} / \mathrm{min}$. The failure load was recorded. SPSS software was employed for statistical analysis. Mean compressive strength and mean compressive load was calculated. One way analysis of variance (ANOVA) followed by post hoc tests served for comparison of compressive strength among the study groups. The significance level was set at 0.001 .

Conclusion: Tetric $\mathrm{N}$ ceram has a high compressive strength compared to amalgomer $\mathrm{CR}$ and equia forte. It can be concluded that tetric $\mathrm{N}$ ceram may be a better posterior restorative in comparison with Amalgomer CR and Equia Forte.

Keywords: Amalgomer CR, Compressive strength, Equia Forte, Posterior class II restorations, Tetric N Ceram.

How to cite this article: Baby S, Ummar A, Mathew J, George L, Paul S. Comparative Study on the Compressive Strength of a New Ceramic Reinforced Glass lonomer (Amalgomer CR) and Resin-coated High Strength Glass Ionomer Cement (Equia Forte) with a Nanohybrid Composite Material (Tetric N Ceram) in a Simulated Oral Environment: An In Vitro Study . Cons Dent Endod J 2018;3(2):40-44.

\footnotetext{
${ }^{1,2}$ Postgraduate Student, ${ }^{3}$ Professor, ${ }^{4}$ Professor and HOD, ${ }^{5}$ Senior Lecturer

${ }^{1-5}$ Department of Conservative Dentistry and Endodontics, Annoor Dental College, Ernakulam, Kerala, India
}

Corresponding Author: Sonu Baby, Postgraduate Student, Department of Conservative Dentistry and Endodontics, Annoor Dental College, Ernakulam, Kerala, India, e-mail: sonubaby88@ gmail.com
Source of support: Nil

Conflict of interest: None

\section{INTRODUCTION}

Dental restorative materials which substitute the lost tooth structure should be stable in different oral environment conditions. Degradation of the materials in saliva leads to deterioration and disintegration of the material in the course of time limiting the longevity of the restorative material. Higher failure rates of restorations in class II cavities can be attributed to the role of mechanical stresses due to occlusal loading leading to cuspal flexure. ${ }^{1}$

Recently, a new ceramic reinforced glass ionomer (amalgomer CR) and resin coated high strength GIC (equia forte) has been introduced to the dental market. These tooth-colored products are claimed to be superior in withstanding masticatory load than conventional composite.

Even now, there are concerns regarding strength of glass ionomer cements. More recently, amalgomer CR, a ceramic reinforced GIC has gained popularity on the grounds of better mechanical properties. However, there is no evidence regarding long term mechanical properties like compressive strength of this material. Together with this, there is no information regarding nature of reinforcement of Amalgomer CR.

Moisture is integral to the setting of Glass Ionomer cement (GIC), especially for conventional acid-base reaction GIC (C-GIC). Water is the essential reaction medium and it hydrates the siliceous hydrogel facilitating formation of polyacid salts. ${ }^{2}$ Therefore, mechanical properties of GIC depend on water balance, i.e. the uptake and release of water during storage and manipulation. C-GIC is sensitive to both hydration and desiccation during initial setting. Desiccation retards the setting reaction decreasing strength; shrinkage and crazing also may happen. Hydration or water uptake during setting may compensate for setting shrinkage, but causes wash out of calcium and aluminum ions retarding setting and decreasing surface integrity. ${ }^{3}$ 
All restorative materials serve in warm, moist oral environment. Such an aggressive environment will alter mechanical properties of materials affecting stability of restorations. This makes it very important to study the changes in mechanical properties like strength, hardness, wear resistance, solubility, etc. of restorative materials in simulated oral environment.

\section{AIM}

To compare and evaluate the compressive strength of a new ceramic reinforced glass ionomer (amalgomer CR) and resin coated high strength GIC (equia forte) with a Nano hybrid composite (tetric N ceram)

\section{MATERIALS AND METHODS}

Twenty four maxillary premolar teeth were selected. The teeth were cleaned, immersed in $\mathrm{NaOCl}$ for 5 minutes and stored in physiologic saline at room temperature.

Samples were mounted on an acrylic block (Fig. 1) and class II cavities were prepared with a width of $1 / 3$ rd the intercuspal distance and a depth of $1 \mathrm{~mm}$ for the occlusal step and $1.5 \mathrm{~mm}$ for the proximal box (Fig. 2).

\section{Sampling}

Samples were divided into three groups of 8 teeth each (Fig. 3).

- Group 1: Class II cavity restored with tetric N ceram composite (control group)

- Group 2: Class II cavity restored with amalgomer CR

- Group 3: Class II cavity restored with equia forte Restored samples were stored in artificial saliva for 2 weeks, then subjected to compressive strength testing using the universal testing machine (Instron 3300 universal testing system, North America) at a crosshead speed of $0.5 \mathrm{~mm} / \mathrm{min}$. The failure load was recorded.

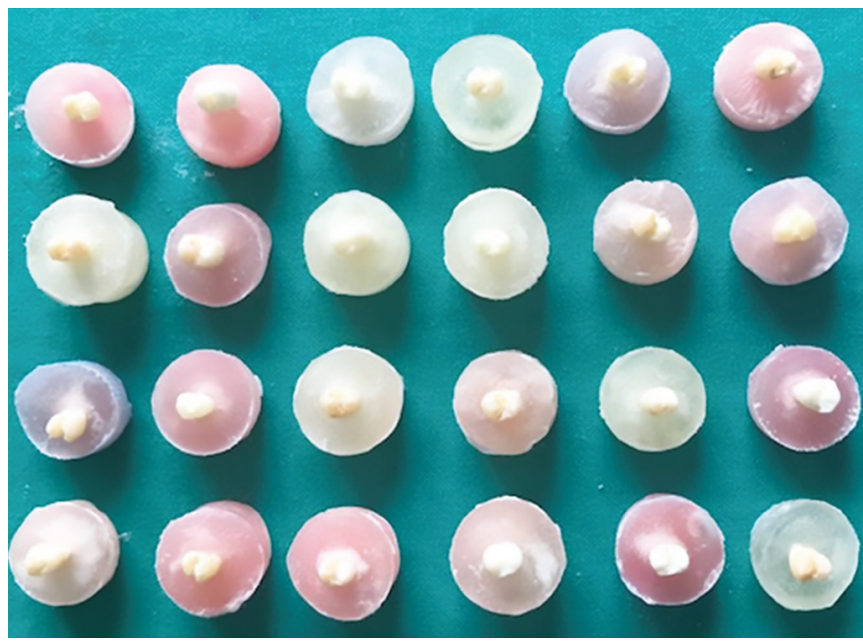

Fig. 1: Mounted samples with the coronal part of the tooth exposed

\section{Statistical Analysis}

Statistical Package for Social Sciences (SPSS) software was used for statistical analysis. Mean compressive strength and mean compressive load was calculated. One-way ANOVA and post-hoc tests served for comparison of compressive strength among the study groups. The significance level was set at 0.001 .

\section{RESULTS}

Mean compressive strength and mean compressive loads are shown in Table 1. Group I showed highest mean compressive strength followed by group I followed by group III. Mean compressive load also followed the same pattern.

The mean compressive load and mean compressive strength between the three different materials were assessed using one-way ANOVA. There was a statistically significant difference in mean compressive strength and load among all the groups $(\mathrm{p}<0.001)$

Pair wise comparison using scheffe post-hoc test (Table2), showed statistically significant difference in mean compressive strength and load between Amalgomer CR and

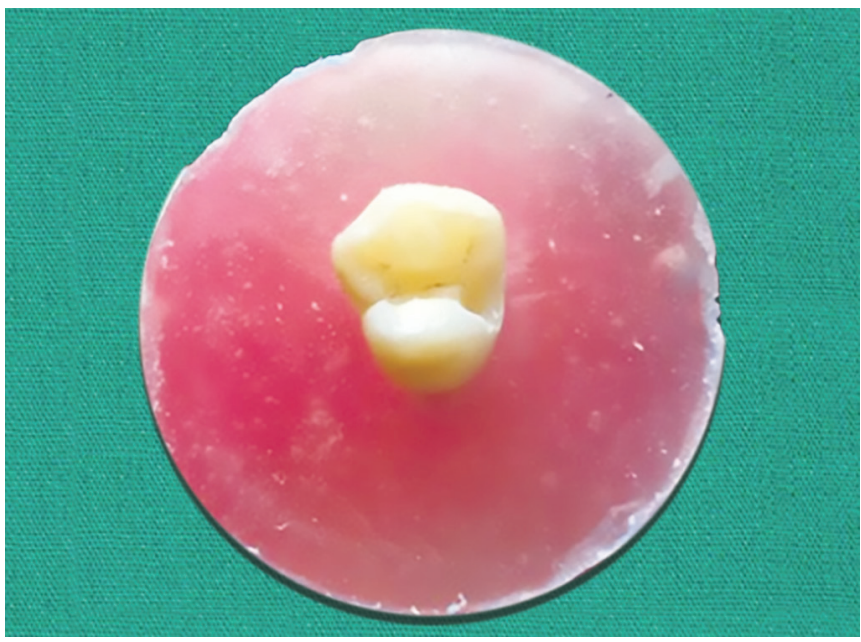

Fig. 2: Class II cavity prepared in a selected tooth

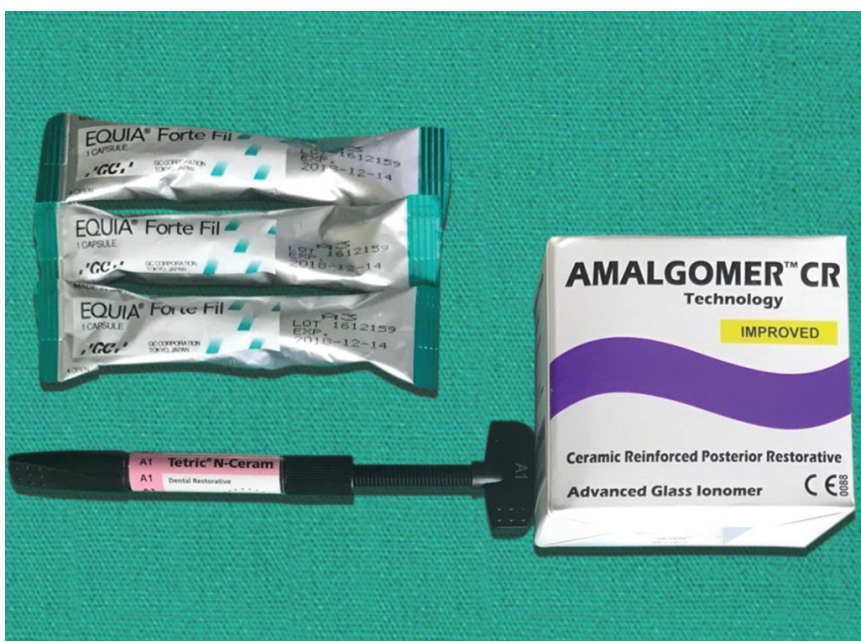

Fig. 3: Materials used for study. (a) Equia Forte; (b) Tetric N Ceram; (c) Amalgomer CR 
Table 1: Results of one way ANOVA analysis

\begin{tabular}{llllllll}
\hline \multicolumn{7}{c}{ One-way ANOVA } & \\
\hline MCL & & $N$ & Mean & Sd & $F$ & $D f$ & $p$ \\
Composite & 8 & 8 & 1848.024 & 190.085 & 13241 & 23 & $<0.001$ \\
Equia & 8 & 8 & 2467.363 & 547.099 & & & \\
\hline Total & 24 & 8 & 1391.830 & 439.042 & & & $<0.001$ \\
\hline MCS & Amalgomer & 8 & 1902.406 & 602.892 & & 23 & \\
Composite & 8 & 8 & 29049.107 & 2987.952 & 13.241 & & \\
Equia & 8 & 8 & 38784.507 & 8599.867 & & & \\
\hline Total & 24 & 24 & 21878.202 & 6901.313 & & & \\
\hline
\end{tabular}

Table 2: Post-hoc test, significant difference seen between the groups

\begin{tabular}{lllll}
\hline \multicolumn{5}{c}{ Scheffe post-hoc test } \\
\hline Dependent Variable & Reference group & Comparision group & Mean difference & $p$ \\
\hline \multirow{3}{*}{ MCL } & \multirow{2}{*}{ Amalgomer } & Composite & $-619.33894^{*}$ & 0.026 \\
& & Equia & 456.19343 & 0,119 \\
\cline { 2 - 5 } & Composite & Equia & 1075.53237 & $<0.001$ \\
\hline \multirow{3}{*}{ MCS } & \multirow{2}{*}{ Amalgomer } & Composite & $-9735.40003^{*}$ & 0.026 \\
& & Equia & 7170.90405 & 0,119 \\
\cline { 2 - 5 } & Composite & Equia & $16906 \_30408^{*}$ & $<0.001$ \\
\hline
\end{tabular}

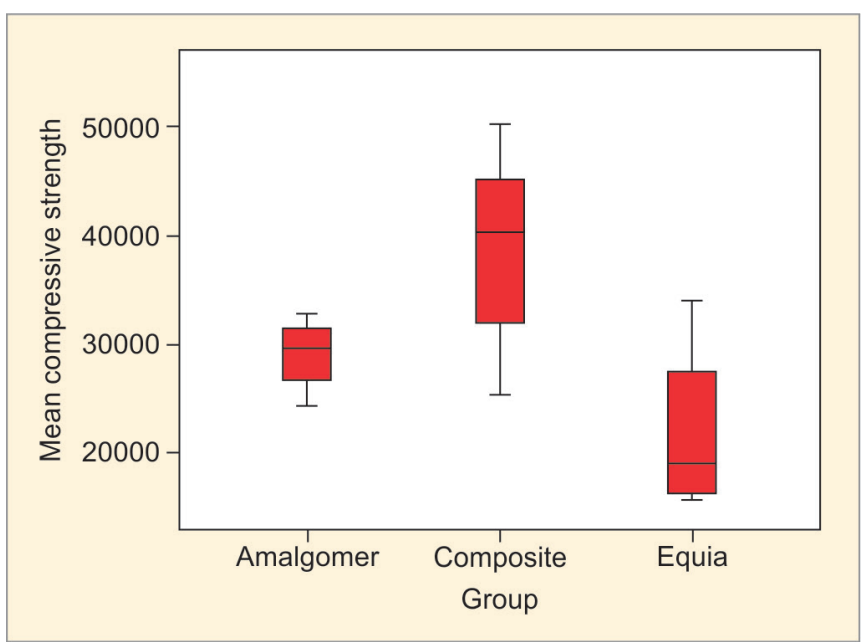

Graph 1: Comparing the mean compressive strength between the groups

composite $(p=0.026)$ and also between composite and EQUIA ( $p<0.001$ ) but there was no significant difference between Amalogomer and equia forte $(p=0.119)($ Graph 1$)$.

\section{DISCUSSION}

In this study, compressive strength obtained was highest for tetric $\mathrm{N}$ ceram followed by amalgomer $\mathrm{CR}$ and least for equia forte. A significant difference in compressive strength was observed between the three groups except for amalgomer $\mathrm{CR}$ and equia forte.

Compressive strength testing is important in in vitro studies since it is considered as a good indicator for simulating the forces that the restorative materials are subjected to under mastication. Compressive strength is measured using the Instron Universal testing machine, as it is a simpler way to analyze the compressive strength. ${ }^{2}$
Artificial saliva was chosen to simulate the natural environment conditions of the oral cavity, though, exact duplication of of human saliva is impossible due to the inconsistent and unstable nature of saliva. Its use is well justified in other in vitro studies. ${ }^{4}$

In this study the tetric $\mathrm{N}$ ceram showed the highest compressive strength. The combination of a nanohybrid with the prepolymer technology, is used in tetric $\mathrm{N}$ ceram which is composed of prepolymerized and milled microfillers, ytterbium fluoride particles and nanofilers. ${ }^{5,6}$ These nanohybrid composites have high filler content and monomers which imparts better mechanical properties and surface finish.

According to manufacturers tetric $\mathrm{N}$ ceram is made of Bis-GMA, Bis-EMA and UDMA and show low volumetric polymerisation shrinkage. ${ }^{7}$ Tetric $\mathrm{N}$ ceram is produced by coordinated and optimised mixing of monomer matrix and fillers. 8,9

Ytterbium fluoride added to obtain high radiopacity also releases fluoride. Spherical mixed oxide particles reduces wear and provides favourable consistency. ${ }^{3}$ They also minimize the thickening effects of fillers, as they provide large volume with the smallest surface area possible..$^{10}$ Primary particles, (individual bodies) are combined to secondary particles (agglomerates) to achieve the ideal consistency. ${ }^{3}$

A patented special filler, partially functionalized by silanes, performs as an unique shrinkage stress reliever, reducing the shrinkage stress of tetric $\mathrm{N}$ ceram to minimum level. ${ }^{7,11}$ Reduced polymerisation shrinkage leads to lower volumetric shrinkage, improves marginal integrity and reduces shrinkage stress over the composite 
surface and on the adhesive bond..$^{12}$ Newly added light initiator Ivocerin-a dibenzoyl germanium derivative facilitate application and curing of larger increments of up to $4 \mathrm{~mm}$, without adversely affecting the optical properties such as translucency or colour. ${ }^{13}$

Recently, introduced amalgomer CR (ceramicreinforced glass ionomer) combines the high strength of a metallic restorative, esthetics and other advantages of glass ionomers, which not only complies with the international standards of GIC but with the standard for amalgam. ${ }^{14}$ The ceramic also helps in imparting excellent wear and erosion resistance and also enhances the radiopacity and all-round strength of the cement. ${ }^{15}$

Ayad et al. determined the compressive strength (CS), diametric tensile strength (DTS), surface hardness (SH), and surface roughness (SR) of Amalgomer CR in comparison to high-copper dental amalgam. ${ }^{2}$ It was concluded that the physicomechanical properties of the tooth-colored ceramic reinforced glass ionomer were so close and sometimes significantly superior to dental amalgam. ${ }^{16}$

Setting mechanism of amalgomer $\mathrm{CR}$ is like conventional acid-base reaction GIC. The material has a particulate ceramic component that increases its strength, with other general characteristics of GIC. Gu, in his study, stated that zirconia is the most important crystalline ingredient of amalgomer CR. Zirconia is added for strengthening and toughening of some composites because it undergoes a phase transformation from tetragonal to monoclinic when subjected to stress. ${ }^{8}$ This change in phase causes a $4 \%$ change in volume producinga local compressive stress, which counteracts crack opening tension, inhibiting crack propagation leading to increased fracture resistance. ${ }^{17}$ This increase in fracture resistance prompted its use in GIC, although there is no evidence to prove that it would behave in the same manner in the more ductile matrix. The manufacturer claims that the partial reaction of ceramic filler with the matrix produce some bonding and also alters the polysalt matrix. ${ }^{18}$

Equia forte (GC), a new glass ionomer restorative system, is a combination of a self-adhesive, chemically cured, highly filled GIC (Fuji IX GP Extra, GC) and a selfadhesive, light cured, filled resin surface sealant (G-coat Plus, GC). It is claimed that the material has increased fracture toughness, flexural strength, and flexural fatigue resistance. ${ }^{19}$ The surface coating agent of the equia system is made of a nanofilled resin that significantly increases the resistance of the material to mechanical forces. Adhesion of equia to dentin occurs by a micromechanical interlocking and a chemical bonding with the hydroxyapatite in enamel and dentin. ${ }^{20}$

\section{CONCLUSION}

In this study, tetric $\mathrm{N}$ ceram showed the highest compressive strength in comparison to amalgomer $\mathrm{CR}$ and equia forte. Amalgomer CR and equia forte showed similar results. It can be concluded that tetric $\mathrm{N}$ ceram may be a better posterior restorative in comparison with amalgomer $\mathrm{CR}$ and equia forte.

\section{REFERENCES}

1. Vaid DS, Shah NC, Bilgi PS. One year comparative clinical evaluation of EQUIA with resin-modified glass ionomer and a nanohybrid composite in noncarious cervical lesions. Journal of conservative dentistry: JCD. 2015 Nov;18(6):449-452.

2. Ayad NM, Elnogoly SA, Badie OM. An in-vitro study of the physico-mechanical properties of a new esthetic restorative versus dental amalgam. Archives of Oral Research. 2008 Nov 29;4(3):137-144.

3. Terry DA, Leinfelder KF, Blatz MB. A comparison of advanced resin monomer technologies. Dent Today. 2009 Jul 1;28(7): 122-123.

4. Leung VH, Darvell BW. Artificial salivas for in vitro studies of dental materials. Journal of dentistry. 1997 Nov 1;25(6):475-484.

5. Jain N, Wadkar A. Effect of Nanofiller Technology on Surface Properties of Nanofilled and Nanohybrid Composites. Int J Dent Oral Health, Volume1. 2015;1.

6. Hegde MN, Hegde P, Bhandary S, Deepika K. An evalution of compressive strength of newer nanocomposite: An in vitro study. Journal of conservative dentistry: JCD. 2011 Jan;14(1):36.

7. Tetric SD. Available from URL: http://www. ivoclarvivadent. co. $\mathrm{kr} / \mathrm{ko} /$ products/restorative-materials/composites/tetricn-ceram-bulk-fill 2014.

8. Xu HH, Eichmiller FC, Antonucci JM, Schumacher GE, Ives LK. Dental resin composites containing ceramic whiskers and precured glass ionomer particles. Dental materials. 2000 Sep 1;16(5):356-363.

9. Kim KH, Ong JL, Okuno O. The effect of filler loading and morphology on the mechanical properties of contemporary composites. The Journal of prosthetic dentistry. 2002 Jun 1;87(6):642-649.

10. Suzuki S, Leinfelder KF, Kawai K, Tsuchitani Y. Effect of particle variation on wear rates of posterior composites. American Journal of Dentistry. 1995 Aug;8(4):173-178.

11. Agarwal RS, Hiremath H, Agarwal J, Garg A. Evaluation of cervical marginal and internal adaptation using newer bulk fill composites: An in vitro study. Journal of conservative dentistry: JCD. 2015 Jan;18(1):56-61.

12. Ferracane JL. Resin composite- -state of the art. Dental materials. 2011 Jan 1;27(1):29-38.

13. Palaniappan S, Elsen L, Lijnen I, Peumans M, Van Meerbeek B, Lambrechts P. Three-year randomised clinical trial to evaluate the clinical performance, quantitative and qualitative wear patterns of hybrid composite restorations. Clinical oral investigations. 2010 Aug 1;14(4):441-458.

14. Bhattacharya A, Vaidya S, Tomer AK, Mangat P, Raina AA. Evaluation and comparison of physical properties and fluoride release of newly introduced ceramic reinforced glass-ionomer restorative material with other glass ionomer cements-An in vitro study. International Journal of Applied Dental Sciences 2017; 3(4): 486-492. 
15. Nigam AG, Jaiswal JN, Murthy RC, Pandey RK. Estimation of Fluoride Release from Various Dental Materials in Different Media-An In Vitro Study. International journal of clinical pediatric dentistry. 2009 Jan;2(1):1-8.

16. Wang Y, Darvell BW. Hertzian load-bearing capacity of a ceramic-reinforced glass ionomer cement stored wet and dry. dental materials. 2009 Aug 1;25(8):952-955.

17. Gu YW, Yap AU, Cheang P, Khor KA. Effects of incorporation of $\mathrm{HA} / \mathrm{ZrO} 2$ into glass ionomer cement (GIC). Biomaterials. 2005 Mar 1;26(7):713-720.
18. Kasuga T, Yoshida M, Ikushima AJ, Tuchiya M, Kusakari H. Stability of zirconia-toughened bioactive glass-ceramics: in vivo study using dogs. Journal of Materials Science: Materials in Medicine. 1993 Jan 1;4(1):36-39.

19. Zoergiebel J, Ilie N. Evaluation of a conventional glass ionomer cement with new zinc formulation: effect of coating, aging and storage agents. Clinical oral investigations. 2013 Mar 1;17(2):619-626.

20. Gururaj M, Shetty R, Nayak M, Shetty S, Kumar CV. Fluoride releasing and uptake capacities of esthetic restorations. Journal of Contemporary Dental Practice. 2013 Sep 1;14(5):887-891. 